\title{
Nitrogen Doping Position-Dependent Rectification of Spin- Polarized Current and Realization of Multifunction in Zigzag Graphene Nanoribbons with Asymmetric Edge Hydrogenation
}

\author{
LIHUA WANG, ${ }^{1}$ ZIZHEN ZHANG, ${ }^{1}$ JIANGUO ZHAO,${ }^{1}$ BINGJUN DING, ${ }^{2}$ \\ and YONG GUO ${ }^{1,3}$ \\ 1.-School of Physics \& Electronic Science and Institute of Applied Chemistry, Shanxi Datong \\ University, Datong 037009, Shanxi, China. 2.-State Key Laboratory for Mechanical Behavior of \\ Materials, Xi'an Jiaotong University, Xi'an 710049, Shaanxi, China. 3.-e-mail: ybsy_guo@163. \\ com
}

\begin{abstract}
The magnetic and spin transport properties of asymmetric edge-hydrogenated zigzag graphene nanoribbons (ZGNRs) selectively doped with nitrogen atoms were investigated using spin-polarized density functional theory and nonequilibrium Green's function. Results show that the rectifying performance of spin-polarized current with a ratio higher than $10^{5}$ can be modulated by changing the positions of the nitrogen dopant. Complete spin filtering (100\%) and excellent negative differential resistance behaviors were observed in the ZGNR junctions. These doping position-dependent spin transport characteristics were further tested by shifting from the odd-numbered zigzag-shaped $\mathrm{C}$ chains $\left(N_{\mathrm{Z}}\right)$ to the even-numbered $N_{\mathrm{Z}}$ in ZGNRs. This study suggests that adopting a suitable nitrogen doping position could be an effective approach to significantly enhance the rectifying behavior of asymmetric edge-hydrogenated ZGNRs, which are promising materials for multifunctional spintronic devices.
\end{abstract}

Key words: Rectification, spin filtering, negative differential resistance, doping, zigzag graphene

\section{INTRODUCTION}

Graphene, a single layer of graphite, has been the subject of numerous experimental and theoretical investigations because of its high electron mobility and potential applications in several emerging areas. ${ }^{1-8}$ Quasi-one-dimensional graphene nanoribbons have been experimentally obtained by cutting a graphene sheet into nanometer widths. ${ }^{9}$ In particular, zigzag graphene nanoribbons (ZGNRs) have attracted intensive research interest because of their edge magnetism and unique transport properties, ${ }^{10-17}$ which make them potential candidate materials for spintronic devices. Graphene-based spintronic devices contain charge carriers with spin coherence lengths of 1.5-2.4 $\mu \mathrm{m}$ and spin relaxation times of $0.1-0.4 \mathrm{~ns}$ at room temperature. ${ }^{18,19}$

(Received July 26, 2015; accepted November 7, 2015; published online December 1, 2015)
Previous experiments have performed spin injection of different molecular materials into graphene layers at room temperature ${ }^{20}$ ZGNR-based devices exhibit several spin transport properties, such as a spin filtering effect, ${ }^{17}$ spin-polarized current rectification, ${ }^{21}$ switching, ${ }^{22}$ and giant magnetoresistance phenomenon. ${ }^{13,23,24}$ However, spin-polarized transport cannot be achieved in monohydrogen-terminated ZGNRs because of spin degeneracy. ${ }^{25,26}$ This spin degeneracy, in which one spin channel is conductive and the other is insulated, can be broken by various approaches, such as edge modification, ${ }^{27}$ doping with other atoms, ${ }^{28}$ applying external electric fields, ${ }^{29}$ and topologic line defects. ${ }^{30}$ Asymmetric edge hydrogenation is an interesting method in which one edge of the ZGNR is $\mathrm{C}-\mathrm{H}_{2}$ bonded and the other is $\mathrm{C}-\mathrm{H}$ bonded (referred to as $\mathrm{H}_{2}-\mathrm{ZGNR}-\mathrm{H}$ in the text). Molecular dynamics simulations show that the $\mathrm{H}_{2}-\mathrm{ZGNR}-\mathrm{H}$ structure is stable at room temperature. ${ }^{5}$ Researchers have also demonstrated 
that the composition of $\mathrm{C}-\mathrm{H}_{2}$ and $\mathrm{C}-\mathrm{H}$ bond types at the edges of the GNRs can be easily controlled by modifying the chemical potential of hydrogen. ${ }^{31}$ The ground state of the $\mathrm{H}_{2}-\mathrm{ZGNR}-\mathrm{H}$ scheme is a ferromagnetic (FM) semiconductor, ${ }^{26}$ which has been adopted in the design of graphene spintronic devices. ${ }^{21,32,33}$ For instance, Kang et al. ${ }^{28}$ found that the density of states (DOS) distribution of $\mathrm{H}_{2}-$ ZGNR-H contains two peaks near the Fermi energy $\left(E_{\mathrm{F}}\right)$ level. The valence states below $E_{\mathrm{F}}$ belong to the up-spin, whereas the conduction states above $E_{\mathrm{F}}$ correspond to the down-spin. Deng et al. ${ }^{33}$ reported the presence of dual-spin filter effects under finite bias, that is, the up- and down-spin electrons are filtered at the counter-direction bias in $\mathrm{H}_{2-}$ 5(6)ZGNR-H devices. However, most studies have focused on individual components of rectification, spin filtering, and negative differential resistance (NDR) behaviors. Developing multifunctional graphene-based spintronic devices can be used to improve the integration density of molecular circuits in the future and is crucial for the complete fabrication of spintronics.

Chemical doping is an effective method used to modify the electronic properties and expand the applications of GNRs. ${ }^{34,35}$ Wei et al. ${ }^{34}$ experimentally synthesized nitrogen $(N)$-doped graphene in different positions through chemical vapor deposition. The $a b$ initio calculation shows that $N$-doped $\mathrm{H}_{2}-\mathrm{ZGNR}-\mathrm{H}$ displays a spin filter effect and a NDR phenomenon. ${ }^{28,36}$ However, previous studies did not report the influence of $N$-doping sites on the rectifying features of $\mathrm{H}_{2}-\mathrm{ZGNR}-\mathrm{H}$; as such, the performance of these devices (i.e., NDR effect) must be further improved. Based on first-principle calculations, the possible control of the spin current in $\mathrm{H}_{2}-$ ZGNR-H was explored in the present study by changing $N$-doping positions. Interestingly, the rectification properties of ZGNRs-based devices were substantially enhanced after choosing a suitable substitution site. Perfect spin filtering and excellent NDR effects were also observed in the $N$ doped $\mathrm{H}_{2}-\mathrm{ZGNR}-\mathrm{H}$ junction.

\section{MODELS AND METHODS}

The ribbon width of ZGNRs can be characterized by the even and odd numbers of the zigzag-shaped $\mathrm{C}$ chains $\left(N_{\mathrm{Z}}\right)$ parallel to the direction perpendicular to the nanoribbon axis. Figure 1 presents the models used in this study, where $\mathrm{H}_{2}-\mathrm{ZGNR}-\mathrm{H}$ with $N_{\mathrm{Z}}=5\left(\mathrm{H}_{2}-5 \mathrm{ZGNR}-\mathrm{H}\right)$ is the typical example. The dashed rectangle contains two unequal sublattices, namely, A and B. From top to bottom, the carbon atoms are labeled as $1 \mathrm{~A}, 1 \mathrm{~B}, 2 \mathrm{~A}, 2 \mathrm{~B}, \ldots, 5 \mathrm{~A}$, and $5 \mathrm{~B}$. One $N$ atom is considered to substitute one left electrode carbon atom in site $1 \mathrm{~B}(5 \mathrm{~B}$ or $3 \mathrm{~B})$ and one right electrode carbon atom fixed in site $3 \mathrm{~A}$ to investigate the doping position-dependent rectification features of $\mathrm{H}_{2}-5 \mathrm{ZGNR}-\mathrm{H}$. The corresponding models are referred to as $\mathrm{M} 1 \mathrm{E}, \mathrm{M} 2 \mathrm{E}$, and $\mathrm{MC}$, respectively. Each model is divided into three regions: left electrode, right electrode, and scattering region. The super-cell consists of three repeated $\mathrm{H}_{2}-5 \mathrm{ZGNR}-\mathrm{H}$ pristine cells with one carbon atom substituted by one $N$ dopant, as shown in Fig. 1. In these two-probe systems, we constructed the scattering region as one doped super-cell of the left electrode connected with one doped super-cell of the right electrode; subsequently, the left and right electrodes were set as one doped super-cell.

Structure relaxation and electronic transport properties have been analyzed with the Atomistix Toolkit (ATK) package, which adopts non-equilibrium Green's functions combined with density functional theory. ${ }^{37-39}$ In the transport calculations, the core electrons are described by the TroullierMartins norm-conserving pseudopotential, ${ }^{40}$ and a local spin density approximation (LSDA) ${ }^{41}$ is used as the exchange-correlation potential. The equilibrium density matrix (DM) is evaluated through contour integration, whereas the non-equilibrium DM is numerically computed in line with an imaginary part of $0.001 \mathrm{eV}$, which is parallel to the real axis in the complex plane. The geometric structures used are optimized at the periodic boundary condition until the calculated Hellmann-Feynman force on each atom is lower than $0.02 \mathrm{eV} / \mathrm{A}$. A vacuum layer of $12 \AA$ is added to avoid the interaction between adjacent ribbons. A 150-Ry cutoff energy for the real-space grid is further selected, and the $k$ point sampling points are 1,1 , and 100 in the $x, y$, and $z$ directions, respectively. A single-zeta polarized basis set is chosen for all atoms to achieve balance between calculation efficiency and accuracy. The transmission spectra and nonlinear currents through the central scattering region are calculated from the Landauer-like formula ${ }^{42,43}$ :

$$
\begin{aligned}
I_{\sigma}\left(V_{\mathrm{b}}\right)= & (e / h) \int_{\mu_{\mathrm{R}}\left(V_{\mathrm{b}}\right)}^{\mu_{\mathrm{L}}\left(V_{\mathrm{b}}\right)}\left\{T _ { \sigma } ( E , V _ { \mathrm { b } } ) \left[f_{\mathrm{L}}\left(E, V_{\mathrm{b}}\right)\right.\right. \\
& \left.\left.-f_{\mathrm{R}}\left(E, V_{\mathrm{b}}\right)\right]\right\} d E,
\end{aligned}
$$

where $\sigma=\uparrow$ (up-spin) and $\downarrow$ (down-spin), and $f_{\mathrm{L} / \mathrm{R}}(E$, $V_{\mathrm{b}}$ ) is the Fermi-Dirac distribution function of the left or right electrode. The energy region of the transmission spectrum that contributes to current is referred to as the bias window $\left[-V_{\mathrm{b}} / 2, V_{\mathrm{b}} / 2\right] . T_{\sigma}(E$, $\left.V_{\mathrm{b}}\right)$ is the spin-resolved transmission coefficient defined as:

$$
\begin{aligned}
T_{\sigma}\left(E, V_{\mathrm{b}}\right)= & \operatorname{Tr}\left[\operatorname{Im}\left\{\sum_{L_{\sigma}}^{\mathrm{r}}\left(E, V_{\mathrm{b}}\right)\right\} G_{\sigma}^{\mathrm{r}}\left(E, V_{\mathrm{b}}\right)\right. \\
& \left.\times \operatorname{Im}\left\{\sum_{R_{\sigma}}^{\mathrm{r}}\left(E, V_{\mathrm{b}}\right)\right\} G_{\sigma}^{\mathrm{a}}\left(E, V_{\mathrm{b}}\right)\right]
\end{aligned}
$$

where $G^{\mathrm{r}}$ and $G^{\mathrm{a}}$ are the retarded and advanced Green's function matrices of the central region, respectively; $\sum_{\mathrm{L}}^{\mathrm{r}}$ and $\sum_{\mathrm{R}}^{\mathrm{r}}$ are the retarded selfenergy matrices for the left and right electrodes, respectively. 


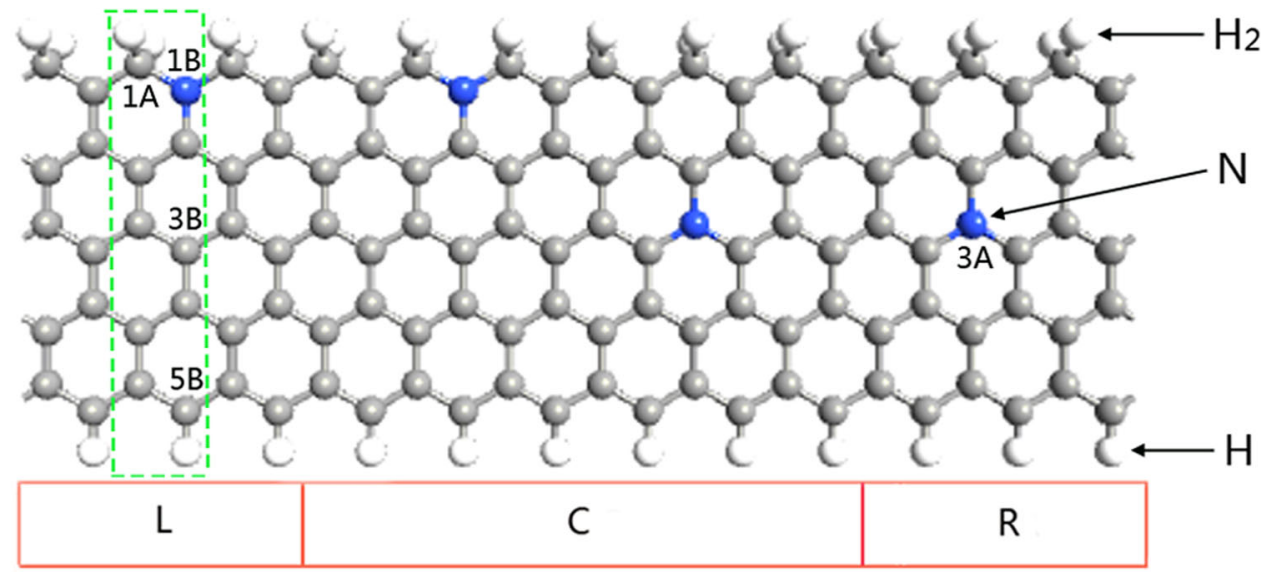

Fig. 1. Two-probe system of $\mathrm{H}_{2}-5 Z$ GNR-H with $N$-doping of both electrodes. $L, C$, and $R$ denote the left electrode, scattering region, and right electrode, respectively. The dashed rectangle contains two unequal sublattices $\mathrm{A}$ and $\mathrm{B}$. From top to bottom, the atoms are labeled $1 A, 1 B, \ldots$. $3 B, \ldots$, and $5 B$. The atoms close to $1 B$ are $1 A$ and $2 A$, and so on.
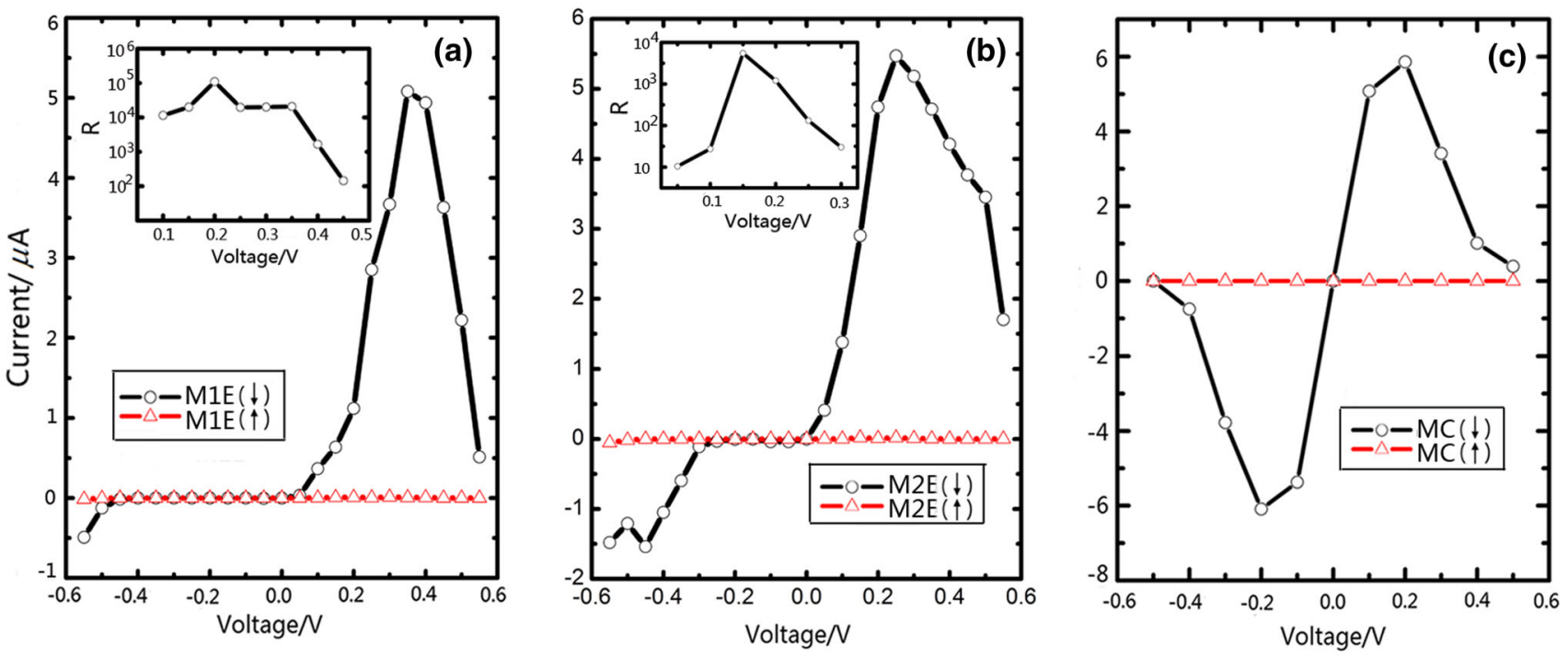

Fig. 2. (a-c) $I-V$ plots of the models (a) M1E, (b) M2E, and (c) MC. The insets in (a) and (b) denote changes in the rectifying ratio $(R)$ of downspin current with applied bias.

\section{RESULTS AND DISCUSSION}

We first inspected the magnetic properties of $N$ doped $\mathrm{H}_{2}-5 \mathrm{ZGNR}-\mathrm{H}$ and considered the following spin configurations: nonmagnetic (NM), FM, and anti-ferromagnetic (AFM) states. When one carbon atom in site $3 \mathrm{~A}$ was substituted by one $N$ atom (green dashed rectangle in Fig. 1), the FM state is the ground state; the total energy of this state is $18 \mathrm{meV}$ per unit cell lower than that of the AFM state and $73 \mathrm{meV}$ lower than that of the NM state. When the carbon atom in the $1 \mathrm{~B}(5 \mathrm{~B}$ or $3 \mathrm{~B}$ ) position was substituted by an $N$ atom, the total energy of the FM state was $22 \mathrm{meV}$ ( $23 \mathrm{meV}$ or $27 \mathrm{meV}$ ) per unit cell lower than that of AFM state and $78 \mathrm{meV}$ $(72 \mathrm{meV}$ or $80 \mathrm{meV}$ ) lower than that of the $\mathrm{NM}$ state. Hence, the FM state is the ground state. We then increased the temperature to $700 \mathrm{~K}$ and compared the results with those above $300 \mathrm{~K}$. The results confirm that the FM state is the ground state. As the FM state is the ground state for $N$ doped of $\mathrm{H}_{2}-5 \mathrm{ZGNR}-\mathrm{H}$, the $[1,1]$ magnetic configuration was chosen to study the spin transport properties of the M1E, M2E, and MC models. The $[1,1]$ magnetic configuration, that is, FM coupling (spins at both edges with the same spin directions) for both electrodes, can be the configuration of the ground state for $\mathrm{M} 1 \mathrm{E}, \mathrm{M} 2 \mathrm{E}$, and $\mathrm{MC}$ under a magnetic field. ${ }^{28,36}$

Figure 2a-c depict the self-consistent calculated currents as a function of the applied bias voltage 

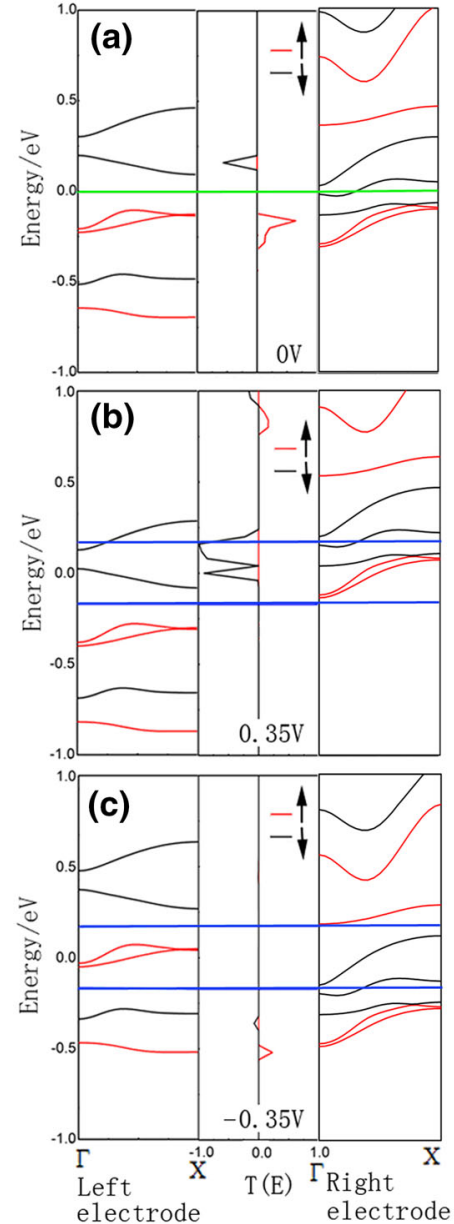
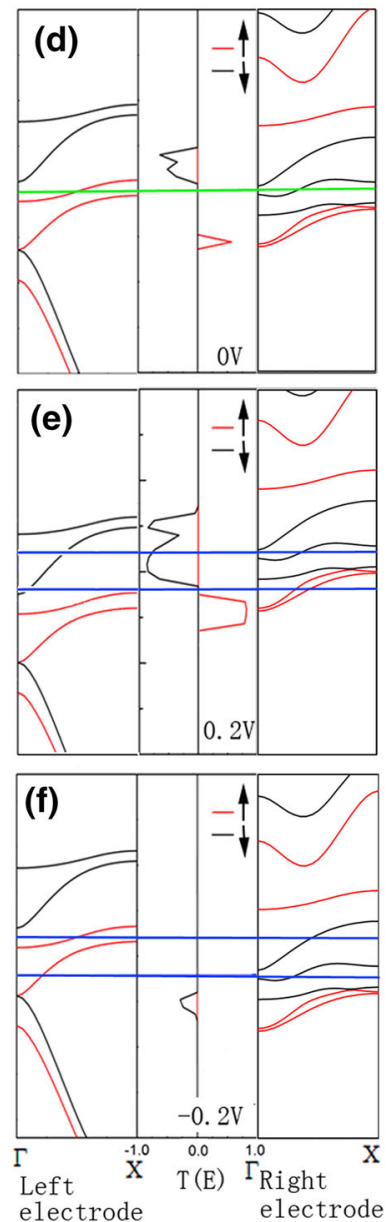
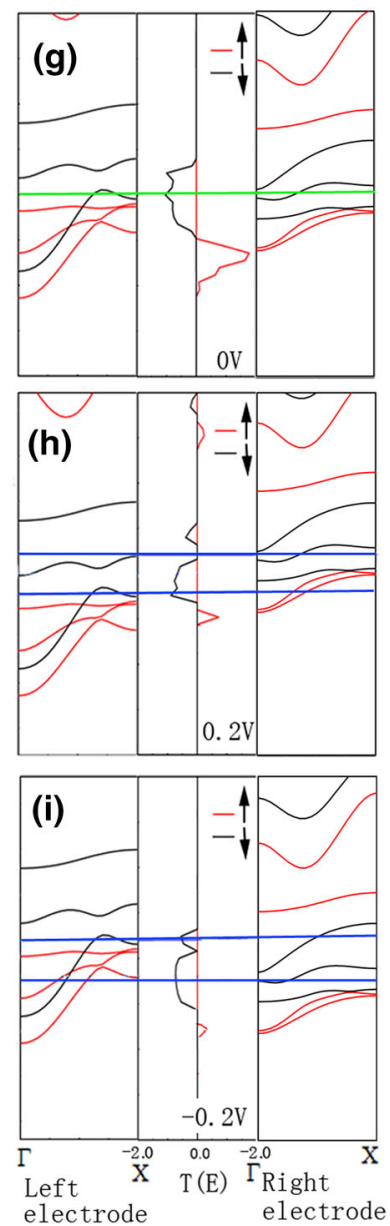

Fig. 3. Band structures for the super-cell of the left electrode (left panels), transmission curve (middle panels), and band structure for the supercell of the right electrode (right panels) for the models (a-c) M1E at $V_{\mathrm{b}}=0.0 \mathrm{~V}, \pm 0.35 \mathrm{~V}$, (d-f) M2E at $V_{\mathrm{b}}=0.0 \mathrm{~V}, \pm 0.2 \mathrm{~V}$, and $(\mathrm{g}-\mathrm{i}) \mathrm{MC}$ at $V_{\mathrm{b}}=0.0 \mathrm{~V}, \pm 0.2 \mathrm{~V}$.

( $I-V$ curves) for the three models. Several distinct features in the evolution of currents can be addressed as follows. First for M1E, the down-spin electrons easily flow through the system at positive bias but are almost forbidden at negative bias. This observation means that the down-spin $I-V$ curve of the model is completely asymmetric, and a strong rectifying behavior is present because the symmetries of the $\mathrm{H}_{2}-5 \mathrm{ZGNR}-\mathrm{H}$ junction are broken by introducing $N$ substitutes. The inset of Fig. 2a shows that the maximum rectification ratios of M1E can reach up to the order of $10^{5}$ when the applied bias is $0.2 \mathrm{~V}$, which is comparable with that of a typical solid-state rectifier. Meanwhile, the current for the down-spin component is larger than that for the up-spin state in the positive-bias region. The corresponding spin polarization is defined as $\left|\left(I_{\uparrow}-I_{\downarrow}\right) /\left(I_{\uparrow}+I_{\downarrow}\right)\right|$ and reaches $100 \%$, which shows a perfect spin filter effect in the positive-bias region for M1E. Furthermore, an interesting NDR phenomenon for the down-spin current is observed in this system in the positive-bias region, with a maximum current at $0.35 \mathrm{~V}$. Second, similar spin transport features are also observed in M2E. However, the maximum rectification ratios can only reach the order of $10^{3}$ at $0.15 \mathrm{~V}$ for the down-spin current, which means that the rectifying behavior of $\mathrm{M} 2 \mathrm{E}$ is lower than that of M1E, as shown in the inset of Fig. 2b. Third, from Fig. 2c, the $I-V$ curve of $\mathrm{MC}$ is quasi-symmetric with respect to $V_{\mathrm{b}}=0.0 \mathrm{~V}$ as evidenced by the quasi-symmetric positions of the $N$ dopants in the left and right electrodes. Consequently, the spin-dependent rectification in this system is negligibly small. Furthermore, the perfect spin filter effect and NDR behavior occur for the down-spin current, regardless of bias polarities, and the peaks for the NDR effect appear at $\pm 0.2 \mathrm{~V}$. And fourth, the spin filter and NDR features are also observed in symmetry-doped $\mathrm{H}_{2}-6 \mathrm{ZGNR}-\mathrm{H},{ }^{36}$ whereas the rectification behavior is not detected. In particular, the performance of the NDR effect in models M1E, M2E, and MC is evidently improved in comparison with symmetry-doped $\mathrm{H}_{2}-6 \mathrm{ZGNR}-\mathrm{H}$.

The bias-dependent transmission spectra and band structures of the super-cells of the left and right electrodes for the models M1E, M2E, and MC 

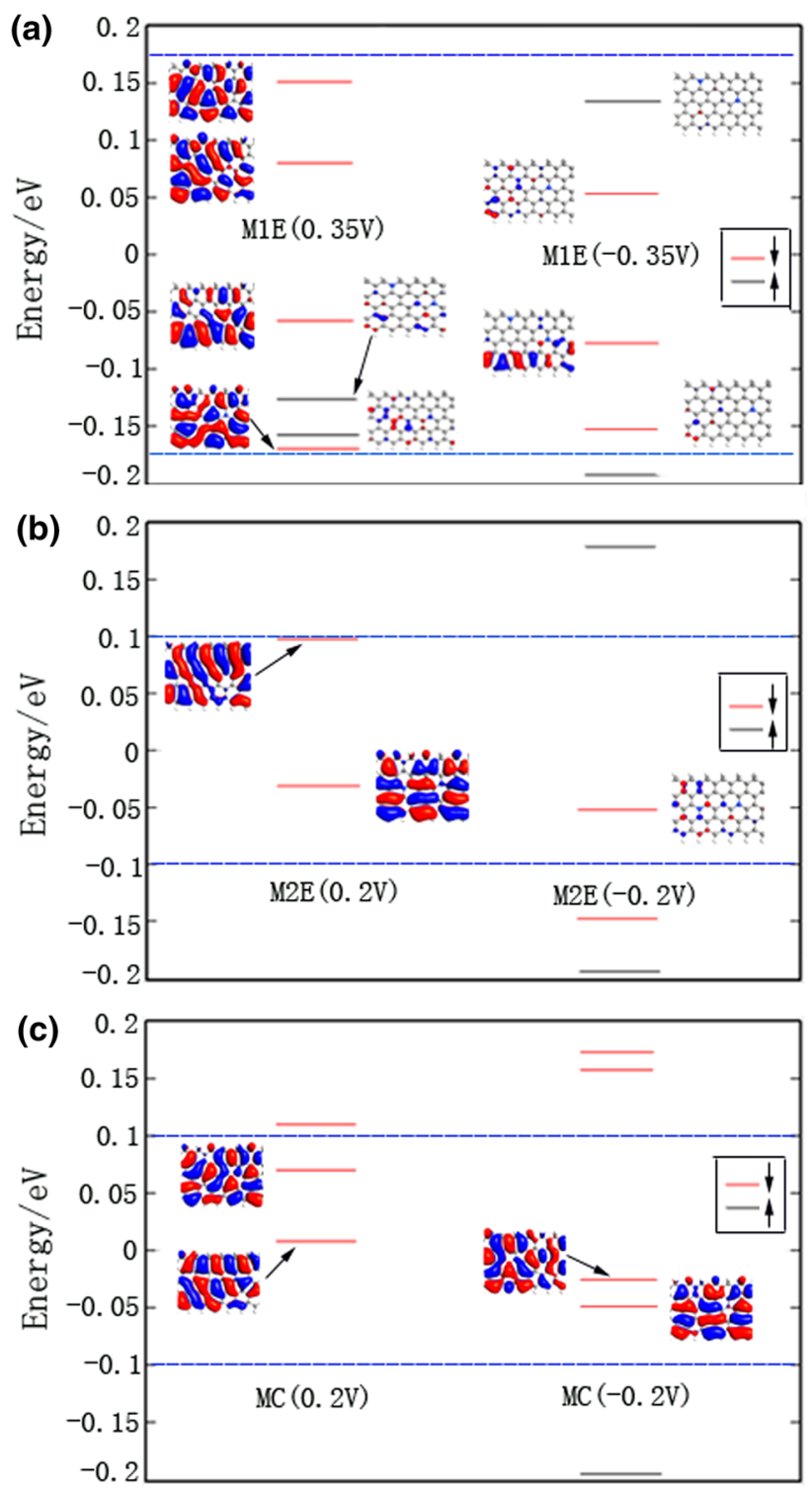

Fig. 4. Energy levels and MPSH within the bias window for three models: (a) M1E at $V_{\mathrm{b}}= \pm 0.35 \mathrm{~V}$, (b) M2E at $V_{\mathrm{b}}= \pm 0.2 \mathrm{~V}$, and (c) $\mathrm{MC}$ at $V_{\mathrm{b}}= \pm 0.2 \mathrm{~V}$. The horizontal dashed lines denote the chemical potentials of the left and right electrodes, and $E_{\mathrm{F}}$ is set to zero.

were determined and shown in Fig. 3 to qualitatively evaluate the $N$-doping position-dependent rectification and spin filter effect in the proposed systems. For the model M1E, the spin band near the $E_{F}$ of the left electrode does not overlap with that of the right electrode under zero bias (Fig. 3a). Electrons transmission between them is also forbidden, thereby causing the transmission coefficients near the $E_{\mathrm{F}}$ to be zero. When the bias is increased to $0.35 \mathrm{~V}$, the energy bands shift downward and upward for the left and right electrodes, respectively. Within the bias window presented in Fig. 3b, only the down-spin bands of the left and right electrodes overlap. Therefore, only the down-spin electron transmission from the left electrode to the right electrode is allowed, resulting in the emergence of down-spin currents but zero up-spin currents. The generated down-spin current is larger than the up-spin current, leading to the perfect spin filter effect in the positive-bias region. When the negative bias is applied, the shift of the energy bands in both electrodes is opposite to that for the positive bias, as shown in Fig. 3c. The down-spin band overlap is not found within the bias window (the same is true for the up-spin band), and no current occurs. The magnitude of the down-spin current at $0.35 \mathrm{~V}$ is significantly larger than that at $-0.35 \mathrm{~V}$, which implies that the rectification behavior is enormous. The significant rectifying effect of the down-spin current should be considered in the model M2E because of the similar bias-dependent transmission spectra and band structure features, as shown in Fig. 3d-f. For the model MC, the upspin band of the left and right electrodes mismatch within the bias window, and no up-spin current is observed. However, the down-spin bands of the left and right electrodes are available and constantly match with each other. These matching down-spin bands contribute to the broad down-spin transmission peak near the $E_{\mathrm{F}}$ shown in Fig. $3 \mathrm{~g}-\mathrm{i}$. Consequently, the total magnitude of the transmission coefficients within the bias window at $V_{\mathrm{b}}=0.2 \mathrm{~V}$ is high and does not differ significantly compared with that at $-0.2 \mathrm{~V}$. The current is the integration of the transmission coefficients in the bias window according to the Landauer-Büttiker formula; ${ }^{42}$ thus, the rectification ratio in $\mathrm{MC}$ is low. Generally, the magnitude of transmission coefficients is mostly related to the number and the delocalization degree of the frontier molecular orbital, which can be obtained from the molecular-projected self-consistent Hamiltonian (MPSH). The MPSH is the selfconsistent Hamiltonian of the isolated molecule in the presence of electrodes but does not include the self-energy of the electrodes. When the applied bias for $\mathrm{M} 1 \mathrm{E}$ is $0.35 \mathrm{~V}$, six energy levels can be observed in the bias window $(-0.175 \mathrm{eV}, 0.175 \mathrm{eV})$. The energy levels include four and two energy levels for the down- and up-spin states, respectively, as shown in the left portion of Fig. 4a. Moreover, the spatial distribution of the corresponding MPSH eigenstates is delocalized for the down-spin and localized for the up-spin. The electronic transport channels are open with large transmission coefficients emerging in the bias window for the downspin or these channels are closed with almost zero transmission coefficients for the up-spin. Therefore, the electronic transmission capability for the downspin is stronger than that for the up-spin. In the right portion of Fig. 4a, three orbitals for the downspin and only one orbital for the up-spin are localized in the bias window at $-0.35 \mathrm{~V}$, thereby suppressing the down- and up-spin of the electronic transport channels. In M2E (Fig. 4b), the spatial distribution of the MPSH for the down-spin state at $\pm 0.2 \mathrm{~V}$ is similar to that of $\mathrm{M} 1 \mathrm{E}$, and no up-spin 
Table I. Local currents of the models M1E, M2E, and MC at $E_{F}$

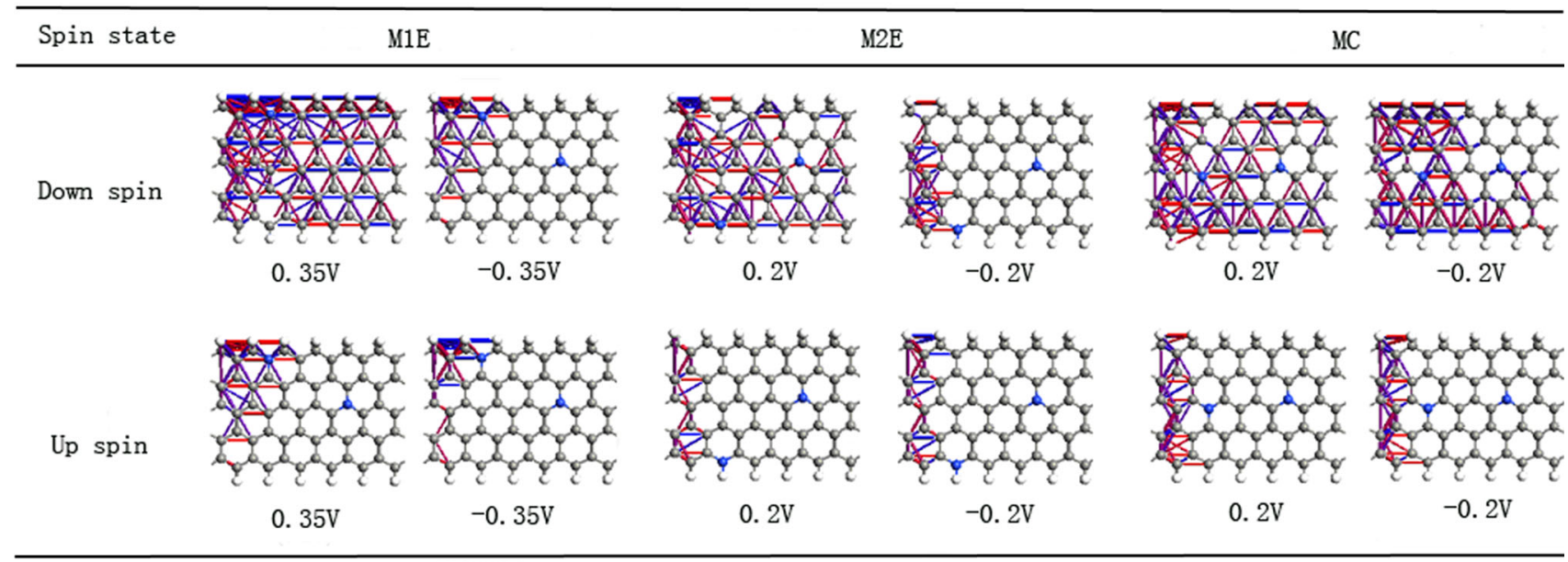
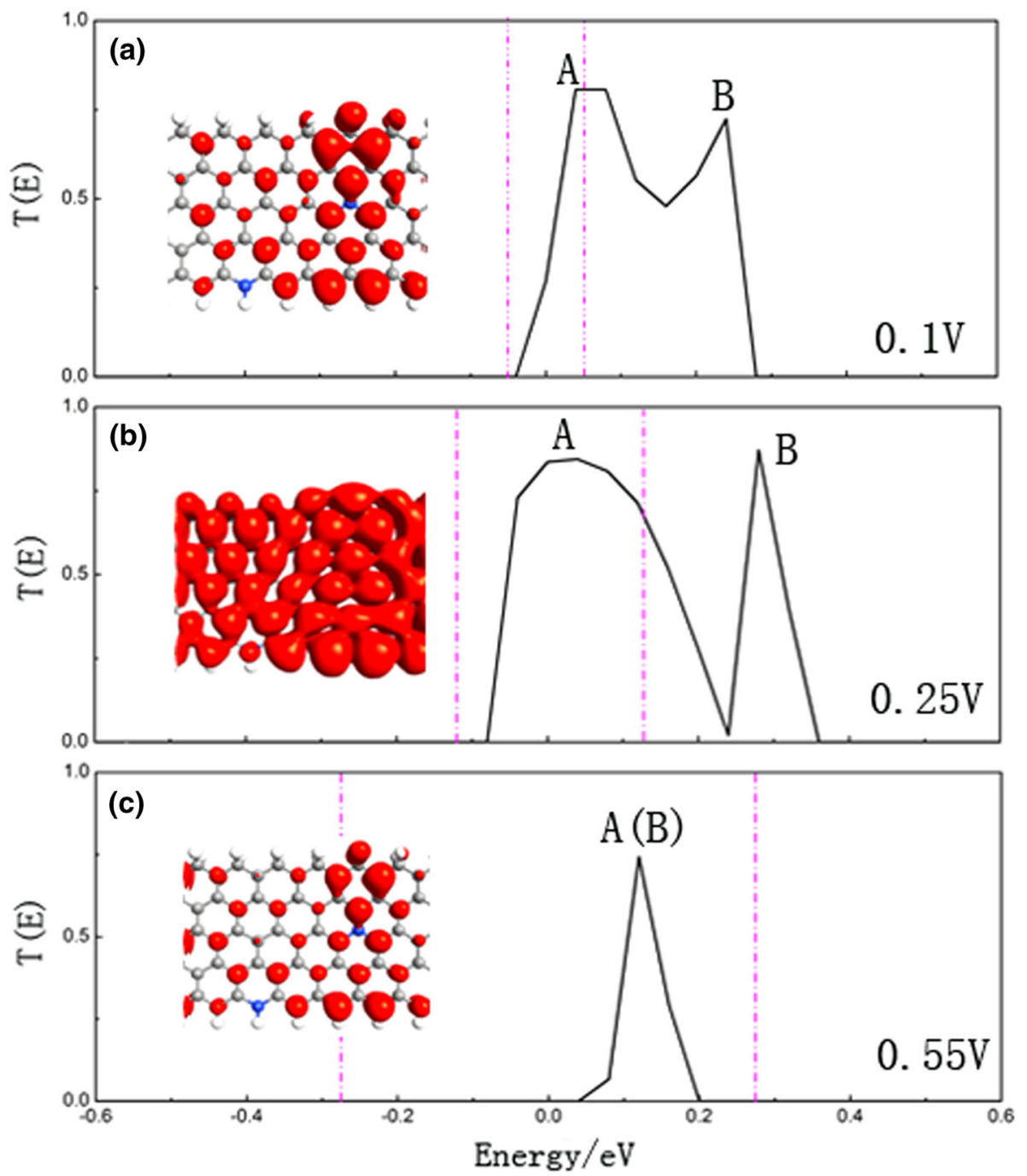

Fig. 5. Down-spin transmission curves (solid lines) of M2E at bias voltages of (a) 0.1 , (b) 0.25 , and (c) $0.55 \mathrm{~V}$. The insets depict the respective down-spin LDOS for the model M2E at $E_{\mathrm{F}}$. The region between the dashed-dotted lines indicates the bias window, and $E_{\mathrm{F}}$ is set to zero. 

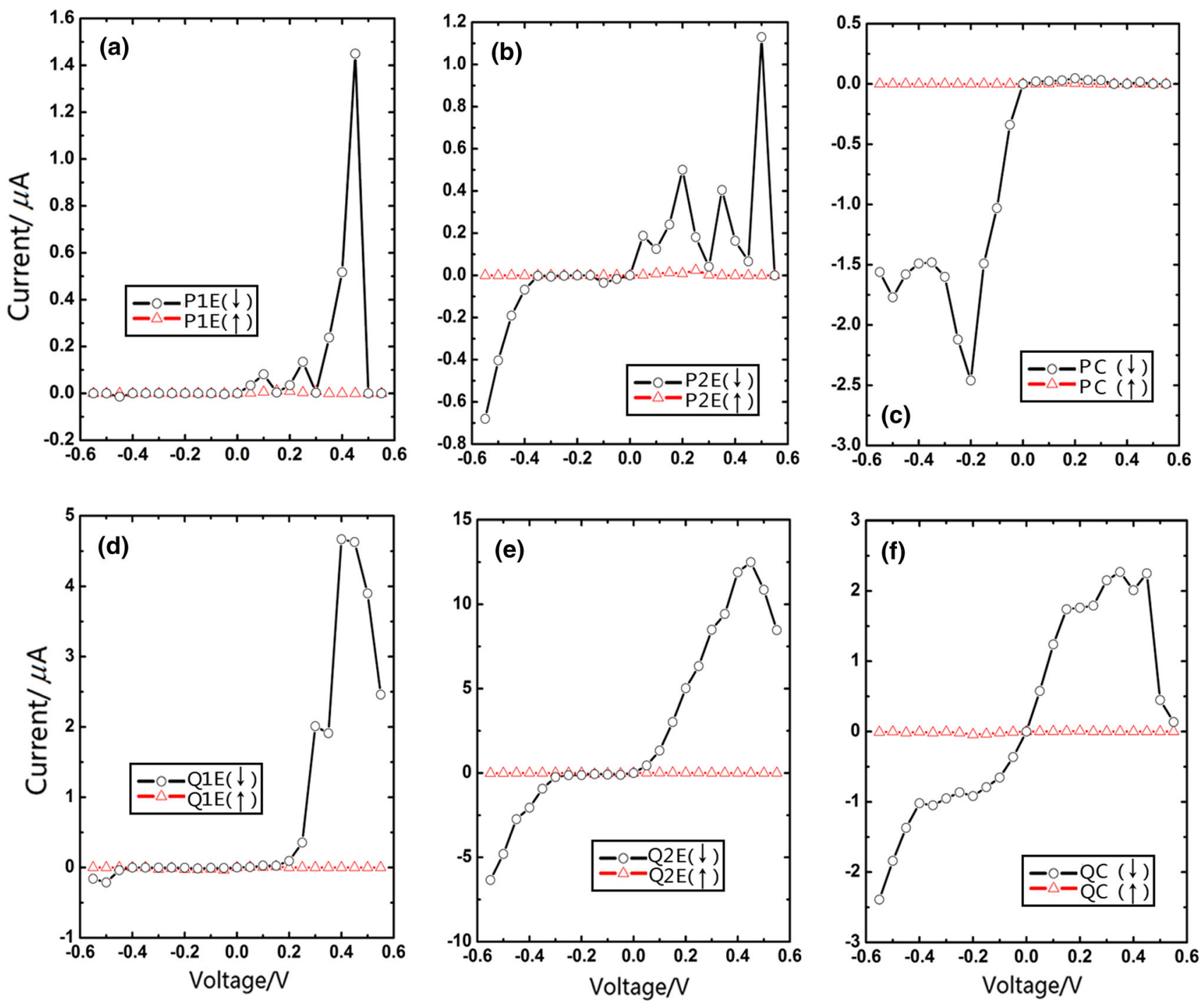

Fig. 6. $I-V$ plots for the up- and down-spin states of the models (a) P1E, (b) P2E, (c) PC, (d) Q1E, (e) Q2E, and (f) QC.

energy level exists within the bias window; thus, only the down-spin current in the low positive-bias region can be observed. By contrast, the spatial distribution of the MPSH for the down-spin state for MC (Fig. 4c) is fully delocalized at $\pm 0.2 \mathrm{~V}$ (no upspin energy level is observed in the bias window), which induces electronic tunneling and contributes to the down-spin current in the positive and negative biases region.

The electron transmission pathways (local current) of the three models in the central region at $E_{\mathrm{F}}$ are plotted in Table I. The electron transmission pathway is an analysis option that splits the transmission coefficient into local band contributions and is commonly used to determine where and how currents propagate. For the model M1E, the up-spin electron transmissions are very weak at $E_{\mathrm{F}}$ at $\pm 0.35 \mathrm{~V}$, and the electrons cannot reach the right electrode. This finding indicates that the current channels for this spin are blocked (Fig. 3b and c) in $E_{\mathrm{F}}$ at which the up-spin transmission coefficient is zero. By contrast, the down-spin electrons can flow through the central region under $0.35 \mathrm{~V}$ and reach another electrode, whereas the opposite trends are observed for $-0.35 \mathrm{~V}$. Generally, two different current channels, namely, chemical bonding and hopping between atoms of the same symmetry sublattice exist in GNR-based devices under bias. ${ }^{6}$ Neto et al. $^{42}$ proposed that electrons in graphene could hop between carbon atoms that belong to the same symmetry sublattice, although the hopping is very weak. However, based on our calculation under applied bias, the hopping current is stronger than the weak bond current, which is similar to that of step-like $\mathrm{ZGNRs}^{4}$ and symmetry-doped $\mathrm{H}_{2}-$ 6ZGNR-H. ${ }^{36}$ In particular, the hopping down-spin currents through M1E occur between carbon atoms with the same symmetry B sublattice, among 

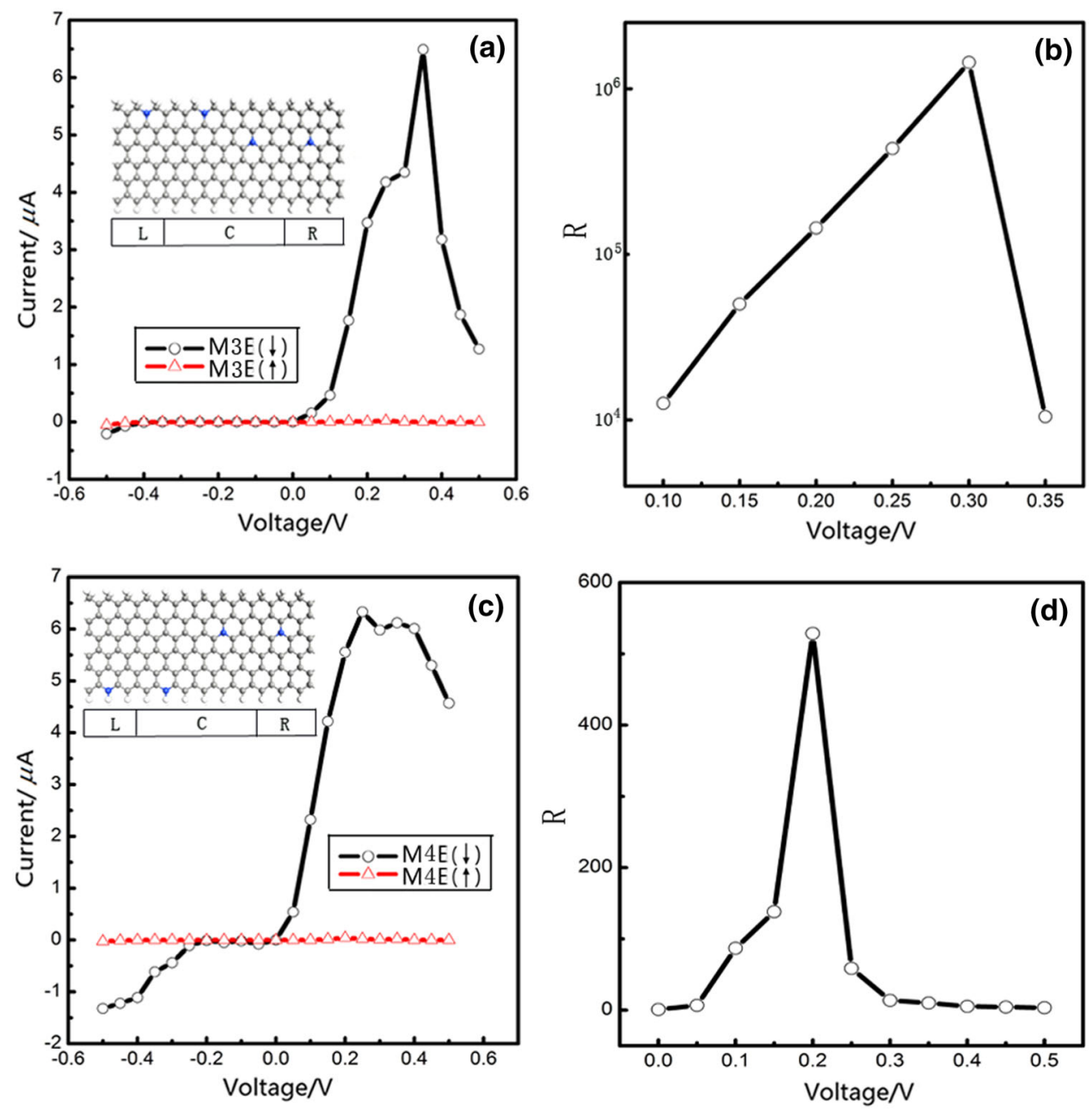

Fig. 7. (a) and (c). $I-V$ characteristics of $\mathrm{H}_{2}-6 Z G N R-H$ with different $N$-doping sites. The insets show the corresponding geometric structures. (b) and (d) Changes in the rectifying ratio $(R)$ of down-spin current with the applied bias for (b) M3E and (d) M4E.

dihydrogen atoms, and between dihydrogen atoms and B sublattice carbon. The down-spin currents can reach the right electrode through the entire ZGNRs. For M2E, the current channel for electrons is similar to that in M1E. The up-spin channels are also blocked at $\pm 0.2 \mathrm{~V}$, and only the down-spin electrons can flow through the down edge of the ribbons and reach the other electrode at $0.2 \mathrm{~V}$. This finding implies that M1E displays stronger downspin electron transmission and greater rectifying behavior than $\mathrm{M} 2 \mathrm{E}$. The transmission pathways further demonstrate that the down-spin currents for MC mainly flow through the edges of ZGNRs at $\pm 0.2 \mathrm{~V}$. The up-spin electron transmissions are also weak and cannot reach the right electrode.

$\mathrm{M} 2 \mathrm{E}$ was chosen as a representative model to explore the details of the NDR effect for down-spin currents in different cases. A series of representative down-spin transmission spectra are shown in Fig. 5, with applied biases of $0.1 \mathrm{~V}, 0.25 \mathrm{~V}$, and $0.55 \mathrm{~V}$. Furthermore, the spatially resolved local densities of states (LDOS) for the down-spin state at
$E_{\mathrm{F}}$ are depicted in the insets of Fig. 5 as a function of the energy of space coordinates. ${ }^{44}$ Two main transmission peaks are observed in Fig. 5 and denoted by A and B. The left peak (A) shifts toward the low energy orientation at $0.1 \mathrm{~V}$, whereas the right peak (B) shows the opposite behavior. The left part of A enters the bias window, thereby inducing a small current growth. Correspondingly, the LDOS of $\mathrm{M} 2 \mathrm{E}$ under $0.1 \mathrm{~V}$ is largely localized in the right component and decreases from right to left, as shown in the inset of Fig. 5a. When the applied bias is further increased to $0.25 \mathrm{~V}$, the LDOS of the down-spin state shows good delocalization, as shown in the inset of Fig. 5b. The contribution of the transmission coefficients within the bias window significantly increases and generates high increases in current, as shown in Fig. 2b. However, the LDOS is highly localized, as shown in the inset of Fig. 5c, and the transmission coefficients in the bias window are reduced when the applied bias exceeds $0.25 \mathrm{~V}$. Such a decrease in the transmission coefficients cannot be compensated by the increased 
magnitude of the bias window. Consequently, the down-spin electronic transfer ability is weakened and the down-spin current is diminished, thereby producing an obvious NDR effect in the positive-bias region.

We also investigated the $I-V$ relationship when the carbon atom of the left electrode in site $1 \mathrm{~B}$ ( $5 \mathrm{~B}$ or $3 \mathrm{~B})$ and the carbon atom of the right electrode at the edge $\mathrm{A}(1 \mathrm{~A}$ or $5 \mathrm{~A})$ are substituted by $N$ atoms. The models corresponding to the doped $1 \mathrm{~A}(5 \mathrm{~A})$ in the right electrode are referred to as $\mathrm{P} 1 \mathrm{E}(\mathrm{Q} 1 \mathrm{E}), \mathrm{P} 2 \mathrm{E}$ (Q2E), and $\mathrm{PC}(\mathrm{QC})$. Figure 6 a depicts the $I-V$ curve of the model $\mathrm{P} 1 \mathrm{E}$, where the down-spin electrons flow through the device in the positive-bias region, but are nearly forbidden under negative bias; thus, the down-spin current presents a rectifying feature. However, the up-spin current is zero in the whole bias range. A spin filter and NDR effect is also present in the positive-bias region. Similar spin transport features are also observed in P2E, Q1E, and Q2E; the only difference among these models is the magnitude of the down-spin current, as shown in Fig. 6b, d, and e. For PC (see in Fig. 6c), whose down-spin current differs from that of $\mathrm{P} 2 \mathrm{E}, \mathrm{Q} 1 \mathrm{E}$, and Q2E, the electrons can flow through only in the negative-bias region. Therefore, the model PC has a perfect reverse rectifying phenomenon. In model $\mathrm{QC}$, the down-spin electrons can flow through almost all the bias region $(-0.55 \mathrm{~V}$ to $0.5 \mathrm{~V})$, and the system appears to have a perfect spin filter behavior between $-0.55 \mathrm{~V}$ and $0.5 \mathrm{~V}$. Furthermore, the rectification of the down-spin current is negligibly small, and the NDR behavior only happens in the positive-bias region.

Given the odd and even numbers of $N_{\mathrm{Z}}$ in ZGNRs, the spin transport results of $\mathrm{H}_{2}-N_{Z} \mathrm{ZGNR}-\mathrm{H}$ with $N_{\mathrm{Z}}=6$ and the geometric structures with different $\mathrm{N}$-doping positions are shown in Fig. 7 , which are called M3E and M4E, respectively. In Fig. 7a and c, the rectifying behavior for the down-spin current and the perfect spin filter effect with $100 \%$ spin polarization can be observed in both systems, as well as an excellent NDR phenomenon for the downspin current in the positive bias region. Meanwhile, Fig. $7 \mathrm{~b}$ and $\mathrm{d}$ show the maximum rectifying ratio higher than $10^{6}$ and $10^{2}$ for $\mathrm{M} 3 \mathrm{E}$ and $\mathrm{M} 4 \mathrm{E}$, respectively. Therefore, the rectifying behavior of M3E is obviously larger than that of M4E. Both models exhibit similar changes in the features of M1E and $\mathrm{M} 2 \mathrm{E}$ (the maximum rectifying ratio higher than $10^{5}$ and $10^{3}$ for M3E and M4E, respectively), as shown in the inset of Fig. 2a and b. These results manifest that the odd and even numbers of $N_{\mathrm{Z}}$ in ZGNRs do not affect the spin transport properties of $\mathrm{H}_{2}-$ $N_{Z}$ ZGNR-H with $N$ dopants. Notably, even when the $p-n$ or $p-i-n$ junctions are not constructed, a large rectification feature can be achieved in ZGNRbased devices by introducing an asymmetric $N$ dopant in both electrodes. Our calculations also show that rectification, spin filtering, and NDR behaviors are observed in $\mathrm{H}_{2}-6 \mathrm{ZGNR}-\mathrm{H}$ when one left electrode carbon atom in site $1 \mathrm{~B}(6 \mathrm{~B}$ or $3 \mathrm{~B})$ and right electrode carbon atom at the edge $\mathrm{A}$ ( $1 \mathrm{~A}$ or $6 \mathrm{~A})$ are substituted by $N$ atoms. These phenomena can be modulated by changing the sites of $N$ dopants in both electrodes.

\section{CONCLUSIONS}

We systematically studied the effect of $N$-doping positions on the magnetism and spin transport properties of the asymmetric edge-hydrogenated ZGNRs by using the non-equilibrium Green's function and density functional theory. The results showed that $\mathrm{H}_{2}-5 \mathrm{ZGNR}-\mathrm{H}$ with asymmetry-doped ZGNR electrodes exhibit a strong rectifying behavior (the maximum rectification ratios can reach the order of $10^{5}$ ), which can be modulated by altering the $N$ substitution sites with a $[1,1]$ magnetism configuration. A perfect (100\%) spin filtering effect in these doped ZGNRs can be observed for the unchanged spin states. Excellent NDR behavior was also detected in the spin-polarized current. Our calculations further showed that the odd and even numbers of $N_{\mathrm{Z}}$ in ZGNRs do not affect the spindependent transport properties of these devices. This study reveals that $\mathrm{N}$-doped $\mathrm{H}_{2}-\mathrm{ZGNR}-\mathrm{H}$ junctions exhibit several useful functions for controlling spin currents.

\section{ACKNOWLEDGEMENTS}

This work was supported by the National Natural Science Foundation of China (Grant Nos. 21301111 and 21503122), Program for New Century Excellent Talents in University (Grant No. NCET-11-1033), Natural Science Foundation of Shanxi Province (Grant No. 2013011007-3), and Datong City Scientific and Technological Project of China (Grant Nos. 201315 and 201422-5).

\section{REFERENCES}

1. F. Prins, A. Barreiro, J.W. Ruitenberg, J.S. Seldenthuis, N. Aliaga-Alcalde, L.M.K. Vandersypen, and H.S.J. van der Zant, Nano Lett. 11, 4607 (2011).

2. A. Pramanik, S. Sarkar, and P. Sarkar, J. Phys. Chem. C 116, 18064 (2012).

3. J. Cai, P. Ruffieux, R. Jaafar, M. Bieri, T. Braun, S. Blankenburg, M. Muoth, A.P. Seitsonen, M. Saleh, X. Feng, K. Mullen, and R. Fasel, Nature 466, 470 (2010).

4. Y.P. An, K.D. Wang, Z.Q. Yang, Z.Y. Liu, G.R. Jia, Z.Y. Jiao, T.X. Wang, and G.L. Xu, Org. Electron. 17, 262 (2015).

5. J. Li, Z.H. Zhang, M. Qiu, C. Yuan, X.Q. Deng, Z.Q. Fan, G.P. Tang, and B. Liang, Carbon 80, 575 (2014).

6. L. Shen, M. Zeng, S. Li, M.B. Sullivan, and Y.P. Feng, Phys. Rev. B 86, 115419 (2012)

7. C.H. Yang, X.Q. Deng, G.P. Tang, and Z.Q. Fan, Solid State Commun. 203, 26 (2015).

8. M. Golor, S. Wessel, and M.J. Schmidt, Phys. Rev. Lett. 112, 046601 (2014).

9. K.S. Novoselov, A.K. Geim, S.V. Morozov, D. Jiang, Y. Zhang, S.V. Dubonos, I.V. Grigorieva, and A.A. Firsov, Science 306, 666 (2004).

10. Y.P. An and Z.Q. Yang, Appl. Phys. Lett. 99, 192102 (2011).

11. D. Gunlycke and C.T. White, Phys. Rev. Lett. 106, 136806 (2011). 
12. L.L. Cui, B.C. Yang, X.M. Li, C. Cao, and M.Q. Long, J. Appl. Phys. 116, 033701 (2014).

13. J. Bai, R. Cheng, F. Xiu, L. Liao, M. Wang, A. Shailos, K.L. Wang, Y. Huang, and X. Duan, Nat. Nanotechnol. 5, 655 (2010).

14. Y. Ni, K. Yao, H. Fu, G. Gao, S. Zhu, and S. Wang, Sci. Rep. 3,1380 (2013).

15. G.P. Tang, Z.H. Zhang, X.Q. Deng, Z.Q. Fan, and H.L. Zhu, Phys. Chem. Chem. Phys. 17, 638 (2015).

16. Y. An, X. Wei, and Z. Yang, Phys. Chem. Chem. Phys. 14, 15802 (2012).

17. M.G. Zeng, L. Shen, M. Zhou, C. Zhang, and Y.P. Feng, Phys. Rev. B 83, 115427 (2011).

18. N. Tombros, C. Jozsa, M. Popinciuc, H.T. Jonkman, and B.J. van Wees, Nature 448, 571 (2007).

19. W. Han and R.K. Kawakami, Phys. Rev. Lett. 107, 047207 (2011).

20. C. Xu, G. Luo, Q. Liu, J. Zheng, Z. Zhang, S. Nagase, Z. Gao, and J. Lu, Nanoscale 4, 3111 (2012).

21. J. Zeng, K.Q. Chen, J. He, X.J. Zhang, and C.Q. Sun, J. Phys. Chem. C 115, 25072 (2011).

22. J. Linder, M. Zareyan, and A. Sudbo, Phys. Rev. B 80, 014513 (2009).

23. W.Y. Kim and K.S. Kim, Nat. Nanotechnol. 3, 408 (2008).

24. F. Munoz-Rojas, J. Fernandez-Rossier, and J.J. Palacios, Phys. Rev. Lett. 102, 136810 (2009).

25. K. Kusakabe and M. Maruyama, Phys. Rev. B 67, 092406 (2003).

26. G. Lee and K. Cho, Phys. Rev. B 79, 165440 (2009).

27. E.J. Kan, Z.Y. Li, J.L. Yang, and J.G. Hou, J. Am. Chem. Soc. 130, 4224 (2008).
28. J. Kang, F.M. Wu, and J.B. Li, Appl. Phys. Lett. 98, 083109(2011)

29. M. Choe, B.H. Lee, G. Jo, J. Park, W. Park, S. Lee, W.-K. Hong, M.-J. Seong, Y.H. Kahng, K. Lee, and T. Lee, Org. Electron. 11, 1864 (2010).

30. B. Xu, J. Yin, Y.D. Xia, X.G. Wan, K. Jiang, and Z.G. Liu, Appl. Phys. Lett. 96, 163102 (2010).

31. T. Wassmann, A.P. Seitsonen, A.M. Saitta, M. Lazzeri, and F. Mauri, Phys. Rev. Lett. 101, 096402 (2008).

32. X.Q. Deng, Z.H. Zhang, G.P. Tang, Z.Q. Fan, and C.H. Yang, Carbon 66, 646 (2014).

33. X.Q. Deng, Z.H. Zhang, C.H. Yang, H.L. Zhu, and B. Liang, Org. Electron. 14, 3240 (2013).

34. D.C. Wei, Y.Q. Liu, Y. Wang, H.L. Zhang, L.P. Huang, and G. Yu, Nano Lett. 9, 1752 (2009).

35. D. Zhang, M.Q. Long, X.J. Zhang, F.P. Ouyang, M.J. Li, and H. Xu, J. Appl. Phys. 117, 014311 (2015).

36. X. Deng, Z. Zhang, G. Tang, Z. Fan, H. Zhu, and C. Yang, Sci. Rep. 4, 4038 (2014).

37. J. Liu, Z.H. Zhang, X.Q. Deng, Z.Q. Fan, and G.P. Tang, Org. Electron. 18, 135 (2015).

38. G.P. Tang, Z.H. Zhang, X.Q. Deng, Z.Q. Fan, Y.C. Zeng, and J.C. Zhou, Carbon 76, 348 (2014).

39. J. Zeng and K.Q. Chen, J. Mater. Chem. C 1, 4014 (2013).

40. N. Troullier and J.L. Martins, Phys. Rev. B 43, 1993 (1991).

41. D.M. Ceperley and B.J. Alder, Phys. Rev. Lett. 45, 566 (1980).

42. R. Landauer, Philos. Mag. 21, 863 (1970).

43. W.J. Liu, S.H. Cai, and X.Q. Deng, J. Electron. Mater. 44, 667 (2015).

44. Y. Cai, A. Zhang, Y.P. Feng, and C. Zhang, J. Chem. Phys. 135,184703 (2011). 